\title{
UNITÉ PROPOSÉE BASÉE SUR LE BRAINSTORMING ASSISTÉ PAR LE MULTIMÉDIA EN VUE DE DÉVELOPPER LES COMPÉTENCES DE LA PRODUCTION ORALE EN FLE AUPRES DES ÉLEVES DU CYCLE PRÉPARATOIRE AUX ÉCOLES DE LANGUES \\ Dr. Heba Attia Ali Salem \\ Maître de conférences en Didactique du FLE \\ Faculté de Pédagogie-Université de Mansourah
}

\section{Introduction:}

La parole se présente souvent comme le moyen de communication le plus important dans la vie quotidienne et sociale. Ainsi, l'enseignement du FLE doit viser à développer auprès des apprenants les compétences de la production orale (parler). Puisque parler une langue étrangère se réalise par des tâches langagières authentiques qui favorisent l'interaction et la communication entre les apprenants, il doit leur donner l'occasion de produire oralement et de parler longuement pour développer leurs aptitudes, leur pensée et leur personnalité. Aussi, par la production orale, ils peuvent recevoir et produire une langue étrangère en l'utilisant d'une manière appropriée et naturelle dans les différentes situations communicationnelles et échanges interculturels.

Malgré l'importance de la production orale pour s'exprimer en FLE et communiquer avec autrui, de nombreux élèves ne peuvent pas bien produire oralement et parler spontanément à cause de manque ou de choix du vocabulaire adéquat, la structure des phrases et la prononciation. Ils parlent seulement en français dans le cours lorsque l'enseignant les interroge. Cet échange ne leur permet pas donc de communiquer en public et de parler la langue française avec aisance et fluidité. Par ailleurs, la peur de commettre des erreurs devant l'enseignant ou les pairs empêche un grand nombre d'élèves d'utiliser leur compétence en production orale dans les diverses situations communicationnelles.

En outre, l'étude du développement de la production orale prend moins de soin de la part de plusieurs chercheurs dans le domaine de didactique du FLE, en dépit qu'il existe une faiblesse en compétences de la production orale chez les apprenants aux différents cycles éducatifs d'après certaines études, citons celles - ci: Sélim (1985), Kassas (1997), Abdel-KHalek (1998), Mohamed (2000), Abdella (2001), Youssef (2003), ElBattrawy (2004), Bakhit (2017).

Ainsi, il y a un besion urgent de différentes études et recherches en didactique du FLE en vue de développer les compétences de la production orale au travers de l'utilisation des techniques d'apprentissage modernes comme le brainstorming et les nouvelles technologies comme le multimédia.

Dans ce cadre, Delacroix (2016) assure que la séance de brainstorming prend la forme d'une réunion de groupe où chacun est invité à émettre librement et spontanément des idées ou suggestions en relation avec le sujet donné. L'intérêt du brainstorming réside dans la quantité des réponses qu'il génère, c'est pourquoi il est important de ne laisser aucun facteur pouvant bloquer la réflexion, la créativité et l'épanouissement des idées, aussi le climat détendu et amical doit encourager les participants à s'exprimer aisément.

Par ailleurs, Guichon (2006) souligne que le multimédia est la cheville 
ouvrière des situations d'apprentissage réussies. Le multimédia et l'enseignement/ apprentissage du FLE sont deux facettes qui doivent interagir ensemble afin de faciliter l'acquisition du français. En effet, l'apprenant pratique peu la langue française lors des cours, le multimédias lui permet de s'entraîner à l'oral, pas seulement en classe, mais aussi en dehors de l'école. Ainsi, chaque établissement scolaire est désormais équipé avec une salle de multimédia.

Par suite, beaucoup d'études ont prouvé l'efficacité du brainstorming et du multimédia sur le développement des compétences de la production orale auprès des apprenants, citons celles - ci: Cullen (1999), Borras (2003), Tomé (2009), Khodadady, Shirmohammadi et Talebi (2011), Liu (2012), Ampa, Rasyid, Basri et al. (2013), Diyyab (2013), Aycan (2013), Agustina et Kumalarini (2014), Asnawi (2015), Ghaemi et Hassannejad (2015), Hamideh, Rostampour et al. (2015).

\section{- Problématique de la recherche}

La problématique de la recherche se résume en faiblesse des élèves de la première année préparatoire aux écoles de langues en compétences de la production orale. Cette faiblesse à cause de ne pas utiliser de nouvelles technologies et de techniques d'apprentissage appropriées au développement des compétences de la production orale auprès de ces élèves.

La chercheuse a constaté cette problématique à travers:

1- Assister à certains cours avec quelques enseignants du français aux écoles officielles de langues à Mansourah où la chercheuse a remarqué que:

A- L'accent est souvent mis sur l'apprentissage de l'écrit. L'oral est relié à l'écrit dans les pratiques enseignantes en classe et peu d'activités de production orale sont effectuées en comparaison d'activités écrites.
B- La majorité des enseignants n'employent pas de nouvelles technologies comme les multimédias qui présentent aux élèves des situations authentiques du français parlé.

C- L'enseignant, c'est lui qui parle le plus en classe et qui corrige les erreurs de ses élèves.

D- Le temps consacré à la prise de parole des élèves est limité. Leurs réponses aux questions de l'enseignant sont fréquemment sous forme d'un mot ou d'une phrase simple.

E- Les élèves n'ont pas suffisamment l'occasion pour s'exprimer et produire oralement soit en continu, soit en interaction.

2- Faire des interviews avec quelques enseignants et orienteurs du français au cycle préparatoire où ils ont assuré les difficultés des élèves à l'oral, ils ne peuvent pas souvent produire oralement de manière efficace et spontanée dans les différentes situations. De plus, la production orale, considérée encore comme la compétence la plus difficile à maîtriser chez plusieurs apprenants du FLE puisque l'enseignement du français dans la classe leur permet peu de pratiquer l'oral.

3- Appliquer un test exploratif à 43 élèves de la première année préparatoire aux écoles officielles de langues à Mansourah en ayant recours à une rubrique de notation pour ce test en vue d'identifier le niveau actuel de leur performance orale. Les résultats de ce test ont indiqué les pourcentages de moyennes des notes d'élèves suivants:

1- Prononcer plus précisement afin d'être facile, compréhensible et confortable de l'autre interlocuteur $(21 \%)$.

2- Posséder un vocabulaire riche et suffisant lors de prendre la parole (28 $\%)$. 
3- Respecter toujours les règles grammaticales pendant la production orale. $(32 \%)$.

4- Parler de façon plus spontanée et plus naturelle (19\%).

5- Produire souvent un énoncé oral compréhensible et approprié à la situation (24\%).

Ainsi, ces résultats prouvent que les élèves de l'échantillon de la recherche ont un manque remarquable en production orale, ils commettent des erreurs d'ordre phonétique, lexical et grammatical et ils ne parlent pas donc avec fluidité. Pour surmonter cette problématique, il faut avoir recours à des nouvelles techniques d'apprentissage comme le brainstorming et le multimédia en vue de développer les compétences de la production orale auprès de ces élèves.

Par suite, cette recherche vise à répondre à ces questions :

1- Quelles sont les compétences de la production orale nécessaires aux élèves de la première année préparatoire aux écoles de langues?

2- À quel point ces élèves possèdent-ils les compétences de la production orale?

3- Quelle est l'efficacité de l'unité proposée basée sur le brainstorming assisté par le multimédia pour développer quelques compétences de la production orale auprès de l'échantillon de la recherche?

\section{- Objectifs de la recherche}

- Identifier les compétences de la production orale nécessaires aux élèves de la première année préparatoire aux écoles de langues.

- Vérifier l'efficacité de l'unité proposée basée sur le brainstorming assisté par le multimédia pour développer quelques compétences de la production orale auprès de l'échantillon de la recherche.

- Faire progresser les apprenants dans la maîtrise des compétences de la production orale de la langue française et leur apprendre à savoir prendre la parole au moment convenable.

- Encourager les enseignants du FLE à prendre soin de la production orale comme objet à travailler en soi et séparé d'autres compétences comme l'écriture et la lecture.

- Faire correspondre le brainstorming comme l'une des meilleures techniques dans l'enseignement/ apprentissage du FLE avec un mélange des nouvelles multimédias pour l'acquisition des compétences orales auprès des apprenants.

- Présenter une variété de communications orales en utilisant des ressources multimédias efficaces en vue de motiver et inciter les apprenants à la participation orale en cours de langue.

- Hypothèses de la recherche

1- Il y a une différence statistiquement significative au niveau 0.05 entre les moyennes des notes d'élèves du groupe de la recherche à la pré/post application du test de la production orale en faveur de la post- application.

2- Il y a une efficacité de l'unité proposée basée sur le brainstorming assisté par le multimédia pour développer quelques compétences de la production orale auprès de l'échantillon de la recherche.

- Délimitations de la recherche

1- Un échantillon composé de 47 élèves en première année préparatoire aux écoles de langues à Mansourah au Gouvernorat d'Al Dakahlia qui apprennent la langue française comme deuxième langue étrangère.

2- Cinq compétences principales de la production orale (la prononciation, le vocabulaire, la grammaire, la fluidité et le contenu) comportant dix souscompétences.

3- Quatre formes du brainstorming: (oral), (individuel), (de groupe), 
(individuel et de groupe)

4- Neuf composantes multimédias: textes illustrés, images, bandes dessinées, CDs, chansons, vidéo-clips, datashow, ordinateur et une affiche colorée concernant les règles $\mathrm{du}$ brainstorming.

- Instruments de la recherche

1- Un questionnaire des compétences de la production orale.

2- Un test de la production orale.

3- Une rubrique de notation pour le test de la production orale (scoring rubric for speaking test).

- Méthodologie de la recherche

La chercheuse a eu recours à deux méthodes:

1- La méthode descriptive qui concerne les principes philosophiques de la recherche (le brainstorming, le multimédia et la production orale).

2- La méthode expérimentale qui concerne l'application d'un test et d'une rubrique de notation pour ce test afin de vérifier l'efficacité de l'unité proposée basée sur le brainstorming assisté par le multimédia pour développer quelques compétences de la production orale auprès de l'échantillon de la recherche.

- Procédures de la recherche

- Afin de répondre à la première question, on a suivi ces procédures:

1- Consultation des ouvrages et des livres qui concernent la production orale en vue d'en profiter pour élaborer un questionnaire des compétences de la production orale.

2- Élaboration d'un questionnaire des compétences de la production orale nécessaires aux élèves de la première année primaire aux écoles de langues.

3- Présentation de ce questionnaire à un jury spécialiste en didactique du FLE pour déterminer les compétences de la production orale les plus nécessaires et convenables à ces élèves.

- Afin de répondre à la deuxième question, on a suivi ces procédures:

1- Élaboration d'un test en vue de détecter à quel point les élèves de l'échantillon de la recherche possèdent les compétences de la production orale.

2- Élaboration d'une rubrique de notation pour ce test (scoring rubric for speaking test) pour vérifier la performance orale des élèves de l'échantillon de la recherche.

3- Présentation de ces deux outils au jury afin de vérifier leur validité.

- Afin de répondre à la troisième question, on a suivi ces procédures:

1- Élaboration de l'unité proposée basée sur le brainstorming assisté par le multimédia en vue de développer quelques compétences de la production orale auprès de l'échantillon de la recherche.

2- Préparation d'un guide pédagogique pour l'enseignement de cette unité.

3- Présentation de l'unité proposée et du guide au jury pour vérifier leur validité.

4- Choix de l'échantillon de la recherche (un seul groupe).

5- Pré- application du test et de la rubrique de notation à cet échantillon.

6- Enseignement de l'unité proposée au groupe de la recherche d'après le brainstorming assisté par le multimédia.

7- Post- application du test et de la rubrique de notation à cet échantillon.

8- Analyse statistique des résultats de la recherche.

9- Interprétation de ces résultats.

10-Proposition de quelques recommandations et suggestions.

- Terminologie de la recherche

- Le brainstorming

Swiners et Briet (2004) définissent le brainstorming comme " une technique de résolution créative de problème sous la direction d'un animateur. C'est plus spécifiquement une réunion informelle de collecte d'idées afin 
d'apporter quelque chose de nouveau dans une structure".

Selon Delacroix et Galtier (2005) le brainstorming est " une méthode participative de résolution de problèmes s'appuyant sur la créativité spontanée des participants. En fait, c'est bien la spontanéité qui est recherchée ".

La chercheuse définit le brainstorming comme une technique d'enseignement qui aide l'apprenant à générer et à produire individuellement ou en groupe des nouvelles idées liées à un sujet donné ou à une question posée en discutant et en échangeant les différents points de vue et idées avec ses collègues sans les critiquer ni les juger afin de choisir avec l'aide de l'enseignant les plus meilleurs et possibles.

\section{- Le multimédia}

Fournier (2005) définit le multimédia comme " un univers informatique qui harmonise en un tout le texte, l'image (fixe et en mouvement) ainsi que le son. Cet univers ainsi créé par la convergence et l'harmonisation de ces médias cherche à transmettre un message, à exprimer des émotions ".

Selon Ngouloure (2013) le multimédia est "l'exploitation simultanée de données sonores, visuelles, informatiques, l'ensemble des techniques de création, de stockage, de transmission, de restitution, et aussi l'ensemble des données permettant cette exploitation simultanée ".

La chercheuse définit le multimédia comme un ensemble de techniques qui transmettent simultanément les informations et les connaissances nécessaires à l'apprenant par des différentes ressources (textes, sons, images, vidéo, CD ou DVD, data-show et ordinateur) et lui permettent de manière interactive de s'exprimer et de communiquer oralement avec les autres.

\section{- La production orale}

Sorez (2003) définit la

production orale comme " transmettre des messages, généralement aux autres, en utilisant principalement la parole comme moyen de communication ".

Selon Georges (2013) la production orale est " capacité à prendre la parole dans une situation directe de dialogue ou d'adresse, reposant sur un authentique engagement énonciatif ".

La chercheuse définit la production orale comme la capacité de l'apprenant à transférer ses connaissances, à exprimer ses idées, ses opinions et ses attitudes de manière bien articulée, structurée, cohérente et fluide dans les différentes situations et ainsi à parler la langue française correctement et avec aisance.

Principes philosophiques de la recherche A- Le brainstorming

La stratégie du brainstorming a été conçue en 1939 par Alex Osborn, les termes «remue-méninges» et «tempête d'idées » sont quelques fois utilisés pour désigner cette stratégie. Celle - ci ne se présente pas comme une technique exclusive pour produire des idées ou une séance courte où les participants trouvent la résolution d'un problème, mais, c'est un processus long comportant un ensemble d'étapes très structurées qui doivent être animées par un professionnel bien entraîné. (Delacroix, 2016)

\section{- Bénéfices du brainstorming}

Le brainstorming joue un rôle essentiel dans la formation de l'apprenant. Il est aussi un outil d'éducation destiné à l'enseignant. Cette activité favorise la fluidité de la pensée de l'apprenant et stimule son aptitude de la concentration et aide au libre flux des idées sans les détruire. De plus, le brainstorming, comme une activité collective ouverte, encourage l'apprenant timide à participer et l'aide à 
être stimulé à écouter de manière attentive et polie les contributions de ses collègues. En exprimant ses points de vue et en écoutant ce que disent les autres, l'apprenant affine sa connaissance ou sa compréhension, en acquérant des nouvelles informations et en accroissant son niveau de conscience. (Swiners et Briet, 2004)

D'autre part, Riot (2013) détermine quelques bénéfices du brainstorming, comme suit :

\section{1- Une étape du processus de décision}

La séance du brainstorming n'est qu'une étape du processus de décision qui commence par déterminer le problème, puis proposer des diverses résolutions et enfin, choisir la meilleure résolution et la mettre en œuvre.

\section{2- Un moyen de transmettre des} connaissances et des compétences

La séance du brainstorming alterne pauses et temps de produire ou d'évaluer les idées. Un transfert des connaissances et des compétences a lieu.

\section{3- Un facteur d'améliorer l'environnement du travail}

Les règles du brainstorming qui contribuent à l'égalité entre les participants, le respect et l'écoute des idées des autres, peuvent améliorer le milieu social dans la classe.

- Principes et règles du brainstorming

Barker (1999) montre que le brainstorming repose sur les principes et les règles suivants:

1) Rechercher le maximum d'idées parce que les meilleures idées peuvent émerger d'une grande quantité que d'une petite. Ces idées seront les chances d'aboutir à des solutions radicales et efficaces.

2) Rechercher des idées originales, aussi les plus folles et les plus absurdes, d'autant plus que des idées étranges ou non-conventionnelles peuvent engendrer d'autres idées réalisables.
3) Pratiquer la méthode des idées-relais à la place de critiquer et de faire écho aux idées d'autrui.

4) Reprendre les idées produites, les étudier et sélectionner les plus meilleures et appropriées.

5) Suspendre le jugement critique. Aucun jugement ne doit être énoncé pendant les étapes de production des idées. Évaluer les idées et non les personnes.

- Formes du brainstorming

D'après (Delacroix et Galtier, 2005),

(Izard et Cahn, 2012), (Leac, 2017), il y a six formes essentielles du brainstorming: 1- Le brainstorming oral (verbal)

Dans cette forme, on peut favoriser essentiellement l'expression imaginaire spontanée. L'apprenant lance une proposition (qui n'est pas encore une idée) et on encourage les autres à produire des associations vagues, des propositions à moitié finies, ce côté vague et informel peut faciliter l'association puisque la proposition est ouverte, et donc accrocher aisément beaucoup de divers concepts.

\section{2- Le brainstorming écrit}

On peut exprimer par écrit une proposition sur un post-it ou un bout de papier, qu'on va lire ensuite à haute voix en même temps qu'on va le coller sur le tableau. On a donc tendance à exprimer des différentes idées réelles ou irréelles. Ces idées écrites favorisent moins l'association collective. En effet, chaque participant peut rebondir sur ces idées et noter sur son post-it des nouvelles idées par association néanmoins, le processus est moins ouvert que dans une procédure verbale.

\section{3- Le brainstorming individuel}

Il a tendance à produire un spectre étendu d'idées en fonction du brainstorming de groupe, cependant il ne développe pas peut-être les idées avec la même efficacité car l'apprenant court fréquemment après le problème qu'il ne peut pas résoudre. Pourtant, l'apprenant mieux explore les idées, sans problèmes de 
temps, sans peur de critique et sans être dominé par d'autres membres du groupe. 4- Le brainstorming de groupe

Il peut développer les idées de manière profonde et effective, car les obstacles que l'apprenant rencontre lors du développement d'une idée, peuvent être surmontés aisément en employant la créativité et les expériences d'autres. De plus, les commentaires des autres sollicitent les idées de chacque participant, mais il a tendance à produire moins d'idées et peut mener à réprimer l'apprenant créatif mais discrèt de la part d'apprenants plus bruyants et envahissants.

\section{5- Le brainstorming individuel et de groupe}

Ils peuvent être combinés : engendrer l'idée principale tout seul, ensuite la faire développer par d'autres dans diverses orientations, par exemple définir d'abord le problème de façon individuelle, et ensuite amener le groupe à proposer beaucoup de résolutions. Celles ci peuvent être améliorées et développées avec le brainstorming individuel.

\section{6- Le brainstorming électronique}

À partir des années 1990, une technique moderne de créativité, qui passe par l'utilisation de l'ordinateur pendant une séance du brainstorming, se développe dans des situations de co-présence ou de distance. Il s'agit du brainstorming électronique. Celui - ci fait travailler les members du groupe chacun sur un ordinateur, en leur demandant d'écrire leurs idées et en leur permettant de voir à l'écran les idées trouvées par les autres members du groupe.

En outre, la simultanéité des communications dans l'environnement électronique peut réduire le processus de (blocage des productions) en général remarqué dans les formes traditionnelles $\mathrm{du}$ brainstorming. Pourtant, il place le participant dans des situations où il répart son attention entre deux activités.

\section{- Comment animer une séance de brainstorming? \\ Selon (Bachelet, 2009),}

(Mesnards, 2012), (Riot, 2013), il existe huit phases fondamentales afin d'animer une séance de brainstorming:

\section{1- Préparer la séance}

Pour que la séance de brainstorming soit efficace, il faut que l'animateur ou les participants s'y préparent. Il doit énoncer clairement le sujet et définir le contexte (quoi, comment, quand, pourquoi...). Par ailleurs, les conditions matérielles sont très importantes: prévoir une salle spacieuse pour favoriser le mouvement, équipée de paperboards et de feutres de couleurs, etc.

2- Introduire la séance

L'entrée en matière est essentielle pour le bon déroulement de la séance : écrire lisiblement l'objectif de la séance sur le paperboard ou sur le tableau et planifier l'agenda (séquences, pauses, fin). 3- Présenter la question de départ

En l'écrivant sur le paperboard, cela permet à tous d'avoir le fil toujours dans leur champ de vision et limite la fluidité d'idées à venir. Il est aussi utile de donner la possibilité aux participants de réagir aux éléments de contextes en limitant le temps à y consacrer.

\section{4- Afficher les règles}

L'éclaircissement des règles du comment faire un brainstorming permet de donner des repères créatifs.

\section{5- Purger les idées existantes}

La purge est un tour de table des idées déjà existantes sur le sujet. Il faut donner 3 à 4 minutes pour noter les idées sur un papier puis faites le tour de table et noter les idées au tableau sans les commenter ni les interpréter. Cette technique permet aux participants de se libérer de leurs premières idées, ils seront ainsi plus disponibles pour en trouver de nouvelles. 


\section{6- Produire le maximum des nouvelles} idées

Une fois la production d'idées lancée, il faut stimuler les participants en permanence par des relances telles que : «Quoi d'autre ?», «Ça vous fait penser à quoi?», «Et encore?».

\section{7- Approfondir les idées}

Cette phase consiste à transformer les idées de la phase précédente en idées finies. Une sélection devra donc être faite.

\section{8- Établir des fiches idées}

Les idées approfondies sont à reporter sur le support de la fiche idée. Un travail en sous groupe de deux à trois apprenants peut alors s'envisager. Il faut leur donner des fiches vierges et leur attribuer une ou deux idées à retranscrire en faisant figurer les éléments suivants : L'objectif de l'idée, son titre, sa description, ses points positifs, les difficultés de son application, le choix : trois possibilités (à retenir, en réserve et à rejeter) et le plan d'action : les premières étapes pour les idées à retenir. Ensuite, il faut terminer par une rapide mise en commun.

- Rôle de l'animateur (l'enseignant) dans la séance du brainstorming D'après (Stoebe et Nijstad, 2003), (Leac, 2017), l'animateur a un rôle principal dans la séance du brainstorming:

- Poser des questions ou des problèmes.

- Faire émerger une grande quantité d'idées permettant l'expression libre.

- Inciter l'apprenant à se mettre en jeu, en exprimant ses idées et ses avis.

- Donner à l'apprenant la chance d'échanger ses idées et d'accroître ses connaissances, en le habituant à construire sur les contributions d'autres.

- Stimuler l'écoute active des apprenants pendant les séances en contrôlant le comportement d'écoute.
- Observer les habiletés des apprenants lors de concentrer sur un sujet ou d'accomplir la tâche du groupe.

- Déterminer le rôle de chaque membre au groupe pour faire participer tous les membres du groupe à exécuter la tâche demandée.

- Créer une atmosphère amicale et positive.

- Rebuter les commentaires d'évaluation ou critiques de l'apprenant pendant la production d'idées.

- Apprécier la participation de l'apprenant dans l'expression orale ou écrite d'idées.

- Faire respecter les procédures et les règles du brainstorming.

- S'assurer que tous ont la parole en veillant à l'égalité du temps de parler : faire taire les bavards et parler les silencieux.

- Organiser la séance de manière pratique (choix du lieu, de l'horaire, des supports et des moyens).

\section{B- Le multimédia}

Le multimédia est un terme qui vient de la langue anglaise et est apparu vers les années 1980 avec le vidéodisque analogique. Au milieu des années 90, il fut le terme retenu par l'industrie de l'informatique en vue de décrire la convergence des applications informatiques. Depuis la fin des années 2000, on utilise le mot multimédia afin de désigner toute application employant à travailler sur au moins un média spécifique. Il est le carrefour de plusieures domaines : audiovisuel, informatique, telecommunications, art graphique, publicité et électronique. (Legros et Crinon, 2002)

- Avantages du multimédia

Le multimédia a beaucoup d'avantages: la facilité du transfert d'information, la durée des leçons est libre et souple. Avec le multimédia, l'apprentissage devient ludique, attrayant 
et créative puisque ce moyen est vivant, divers et favorise la participation et la motivation en suscitant l'envie de la découverte et la continuité, avec des images et des sons. Les apprenants peuvent, grâce au multimédia, écouter la langue originale autant qu'ils le veulent et quand ils le veulent et être en contact avec la culture visée. En outre, l'utilisation du multimédia peut développer des compétences langagières (compréhension ou communication écrite ou orale) et une attitude positive auprès des apprenents envers l'acquisition d'une langue étrangère. Il leur fournit aussi des aides supplémentaires pour construire l'apprentissage et un parcours d'apprentissage personnalisé par rapport à leur niveau et à leurs centres d'intérêts, qui a lieu de façon autonome d'après leur demande. (Hirschsprung, 2005)

- Composantes multimédias

Selon (Seguy, 1999), (Fournier, 2005), (Ngouloure, 2013) les différents composantes ou objets multimédias sont, comme:

\section{1- Les textes}

Le texte est l'un des plus anciens médium de communication et l'un des plus efficaces pour transmettre l'information. Le message textuel doit être adapté aux fins recherchées dans le processus de communication. De plus, il est important, avant de rédiger des textes qui viendront composer le contenu multimédia, de bien cerner les intentions afin d'utiliser le langage textuel approprié.

\section{2- Les images}

En effet, depuis plusieurs années, l'image fixe ou animée, occupe une part croissante de notre vie quotidienne, envahit de plus en plus d'espaces. En outre, les images sont utilisées pour créer des situations qui servent de point de départ pour travailler l'expression orale et écrite. ILs peuvent aussi faciliter l'explication du vocabulaire et des expressions du FLE sans devoir passer par la traduction en langue maternelle.

\section{3- Les sons}

Avec le son, les apprenants sont exposés à des enchaînements prosodiques et ils se familiarisent ainsi avec le rythme, l'intonation et la sonorité du français parlé dans des énoncés complets. Par ailleurs, les documents enregistrés, bien que ces moyens soient très adaptés pour travailler en classe du FLE, surtout au niveau de la compréhension orale et de l'expression orale, puisqu'ils permettent d'enregistrer les productions des apprenants et ensuite de les comparer avec les modèles présentés.

\section{4- La vidéo}

La vidéo permet à l'enseignant d'exposer la vie réelle, la culture, la civilisation des Français et le réel de l'échange verbal, c'est-à-dire écouter et observer le dire et le faire des natifs. Elle aide ainsi à développer la compétence communicative et à enseigner l'acte de parole dans sa triple nature: acte locutif (l'énonciation dans son ensemble), acte illocutif (l'énoncé avec son intention communicative), acte perlocutif (l'effet de l'énoncé sur autrui) en permettant à l'apprenant d'employer tous les signes de l'interaction verbale: verbaux, non verbaux, vocaux et visuels. C'est donc un outil qui rend la classe vivante plus authentique.

\section{5- Le CD ou le DVD}

Les supports privilégiés du multimédia sont le CD ou le DVD. La donnée contenue sur un CD ou DVD est aisément transportable. Ces outils auxiliaires aident l'apprenant à écouter la langue authentique de la langue cible et à se conscientiser son réel. Ils permettent aussi une flexibilité afin d'individualiser le rythme d'apprentissage et informer de manière ludique l'apprenant au travers des canaux auditifs et visuels. 


\section{6- L'ordinateur \\ L'ordinateur est le média moderne} le plus visible de la seconde moitié du $\mathrm{XXe}$ siècle, et sûrement le média qui domine aujourd'hui après la vidéo. L'ordinateur multimédia apparaît aussi comme le moyen le plus versatile, pouvant être utilisé avec efficacité pour travailler des aspects très variés de la langue étrangère comme l'écriture, la compréhension orale, la lecture et l'expression orale. Par ailleurs, il aide l'élève à être l'acteur dans l'environnement électronique, à accéder à la connaissance d'expert, à utiliser les sources d'information du réseau Internet.

\section{- Types du multimédia}

Guichon (2006) classe le multimédia en deux types:

\section{En ligne}

Les utilisateurs peuvent accéder à la ressource multimédia par un réseau (Internet). Cette ressource est donc stockée à distance sur une autre machine que le poste de travail des utilisateurs. Ainsi, le marché du multimédia en ligne est très ouvert et en plein accroissement.

\section{Hors ligne}

Les utilisateurs peuvent accéder à la ressource multimédia par leur machine ou poste de travail sans être connectés à un réseau. Ainsi, le marché du multimédia hors ligne est plus limité et fréquemment plus visé sur quelques secteurs comme celui des jeux vidéo.

\section{- Rôle de l'enseignant lors de l'utilisation du multimédia}

L'enseignant ne devient plus la seul source qui diffuse l'information, mais concepteur des tâches d'apprentissage, accompagnateur ou tuteur en utilisant le multimédia. Vraiment, il doit se questionner sur le parcours à établir parmi les multimédias variés afin que les élèves apprennent de façon autonome, choisir les supports, maîtriser les outils, mettre en place le volet temporel. Il doit aussi les observer pendant un cours en salle multimédia pour les orienter et les aider. En outre, lors d'évaluation, il ne mesure plus seulement les résultats finals, mais aussi les conduites qui ont tracé le parcours. (Carrier, 2000)

D'autre part, Loiseau et Roch (2002) résument le rôle de l'enseignant en points suivants:

\section{1- Au niveau pédagogique}

- Encourager de façon affective les apprenants, particulièrement ceux qui ne s'habituent pas à des TIC.

- Guider les apprenants pendant rechercher les informations pour développer les compétences essentielles de l'apprentissage.

2- Au niveau de la communication

- Inciter les apprenants à créer des communautés en ligne.

- Créer des débats par des forums de discussion ou de vidéoconférence assistée par ordinateur.

\section{3- Au niveau de la création}

- Produire des pages web personnelles et de l'école.

- Orienter l'apprenant lors de créer un journal électronique interactif qui exploite les diverses ressources multimédias.

- Jouer un rôle plus créatif, celui d'auteur multimédia, en créant des activités (hors ligne et en ligne) par utiliser les "programmes d'auteur" ou "systèmes auteurs" qui ne nécessitent aucun savoir de design ni de programmation.

\section{- Multimédia et communication}

Pothier (2003) montre qu'avec le développement de nouvelles formes d'information et de communication, l'oral a pris encore plus d'importance. L'apprenant peut produire, à travers les supports oraux ou visuels (textes, images), des énoncés oraux en mettant en oeuvre les actes de parole nécessaires pour les situations de communication. De plus, la communication multimédia facilite la 
compréhension et l'apprentissage car elle est très similaire à la communication humaine directe (face à face). Dans une conversation, il y a l'interlocuteur (ce qui serait l'équivalent d'une vidéo) qu'on écoute (audio) tout en accompagnant ses paroles accompagnées de gestes et de mouvements du corps (animations). Ainsi, les TICE contribuent à rendre l'apprenant un interlocuteur capable d'employer le français dans les situations de communication variées.

\section{C- Production orale}

La production orale est une compétence que l'élève doit apprendre d'une manière progressive. Il s'agit d'un rapport interactif entre un émetteur et un destinataire ou récepteur. L'émetteur, c'est celui qui produit le message, et le destinataire celui qui écoute afin de comprendre ce que dit l'émetteur. En outre, la connaissance de la production orale communicative se constitue des divers domaines de savoir et savoir-faire liés à la langue, à l'élève, à la vie et au monde. Ainsi, la production orale est une activité propre et personnelle, elle diffère d'un locuteur à un autre, de la même langue. (Sorez, 2003)

\section{- Différence entre la production orale et} l'expression orale

Roux (2003) assure que la parole (la production orale) n'est pas forcément synonyme d'expression orale, malgré les deux termes sont généralement englobés par celui de communication. Production doit être vue comme la simple émission de sons, de mots, de phrases, alors qu'expression sous-entend des énoncés qui impliquent à des degrés divers leur émetteur. La production orale est donc incluse dans l'expression orale, puisque cette dernière exige l'émission de phonèmes organisés, mais qu'elle est loin de la recouvrir entièrement. Le tableau suivant résume simplement la différence entre les deux termes:
Tableau No.1

Différence entre la production orale et l'expression orale d'après Roux 2003

\begin{tabular}{|c|c|}
\hline Production orale & Expression orale \\
\hline $\begin{array}{l}\text { Discours } \\
\text { mémorisé. }\end{array}$ & $\begin{array}{l}\text { Discours spontané, } \\
\text { improvisé. }\end{array}$ \\
\hline $\begin{array}{l}\text { Discours } \\
\text { stéréotypé. }\end{array}$ & Discours libre. \\
\hline Discours collectif. & $\begin{array}{l}\text { Discours individuel } \\
\text { / personnel. }\end{array}$ \\
\hline $\begin{array}{l}\text { Priorité } r a u \\
\text { discours descriptif } \\
\text { (il). }\end{array}$ & $\begin{array}{l}\text { Priorité au discours } \\
\text { impliquant son } \\
\text { émetteur (je). }\end{array}$ \\
\hline Métalangage. & $\begin{array}{l}\text { Langue de } \\
\text { communication. }\end{array}$ \\
\hline Écrit oralisé. & Oral véritable. \\
\hline $\begin{array}{l}\text { Courbe intonative } \\
\text { forcée } \quad \text { et } \\
\text { artificielle. }\end{array}$ & $\begin{array}{l}\text { Courbe intonative } \\
\text { naturelle et } \\
\text { expressive. }\end{array}$ \\
\hline $\begin{array}{lrr}\begin{array}{l}\text { Priorité } \\
\text { norme } \\
\text { langue. }\end{array} & \text { de } & \text { la } \\
& & \\
\end{array}$ & $\begin{array}{l}\text { Priorité au sens } \\
\text { transmis. }\end{array}$ \\
\hline $\begin{array}{l}\text { Réponses à des } \\
\text { questions fermées. }\end{array}$ & $\begin{array}{l}\text { Réponses à des } \\
\text { questions ouvertes. }\end{array}$ \\
\hline $\begin{array}{l}\text { Réponses à des } \\
\text { questions portant } \\
\text { sur du lexique. }\end{array}$ & $\begin{array}{l}\text { Réponses à des } \\
\text { questions portant } \\
\text { sur des énoncés. }\end{array}$ \\
\hline $\begin{array}{l}\text { Communication } \\
\text { uniquement } \\
\text { linguistique. }\end{array}$ & $\begin{array}{l}\text { Communication } \\
\text { totale (c'est-à-dire } \\
\text { faisant appel à tous } \\
\text { les domaines et les } \\
\text { moyens de la } \\
\text { communication). }\end{array}$ \\
\hline $\begin{array}{l}\text { Réseau de } \\
\text { communication } \\
\text { privilégiant la } \\
\text { place et le rôle de } \\
\text { l'enseignant. }\end{array}$ & $\begin{array}{l}\text { Réseau de } \\
\text { communication } \\
\text { privilégiant la place } \\
\text { et le rôle de } \\
\text { l'apprenant. }\end{array}$ \\
\hline
\end{tabular}

\section{- Importance de la production orale}

Une langue est d'abord et avant tout parlée, c'est une habilité servant à communiquer oralement. La production orale est une compétence communicative qui aide à contacter avec des personnes de langue étrangère et provenant de différentes cultures et permet aussi à 
l'apprenant du FLE d'intervenir, entretenir avec les locuteurs natifs, exprimer ses idées, donner son opinion, raconter et participer à un débat. La production orale occupe donc une place prioritaire et privilégiée dans les relations humaines. Pour communiquer dans une langue l'apprenant doit l'acquérir pour la parler correctement en classe et en dehors de celle-ci. Ainsi, elle constitue l'une des étapes essentielles dans l'apprentissage d'une langue étrangère. (Charmeux, 2003)

$$
\text { D'autre part, Blanche- }
$$

Benveniste (2010) montre que la production orale aide l'apprenant à:

- Exprimer ses expériences personnelles et aussi mieux les comprendre.

- Transmettre ses connaissances et échanger ses informations.

- Donner et justifier ses points de vue sur des différents sujets et situations.

- Discuter des idées, des attitudes et des sentiments.

- Comprendre et estimer les expériences des autres.

- Augmenter publiquement sa confiance en soi.

- Comment être bon locuteur?

Selon Giurgiu (2013) toute

personne souhaitant être un bon locuteur doit prendre en compte :

A- Soigner la diction. Les mots doivent être prononcés correctement, leur signification et leur usage maîtrisés. La prononciation ne doit pas être exagérée.

B- Parler et arrêter de parler. C'est la clef non seulement pour être un bon locuteur, mais aussi être un grand animateur ou interviewer. Il est toujours bon d'attendre que l'autre termine son propos avant de parler de nouveau.

C- Le rythme de la parole . Il ne doit pas parler trop rapidement et précipitamment ni trop lentement.
Chaque locuteur, avec le temps, développe un style propre.

De plus, voici quelques stratégies afin de bien parler:

Pense à pourquoi et à qui tu parles et prépare ce que tu veux dire.

Ajuste ta voix en vue d'être compris.

Regarde l'auditoire.

Lève ta main ou prends poliment la parole. Emploie des mots exacts et des gestes adéquats.

Renforce ton message avec des auxiliaires.

- Types et activités de la production orale

D'après (Pacthod et Roux, 1999), (Lafontaine, 2010), les types de la production orale et leurs activités se divisent en deux:

A- Activités de la production orale en continu (texte oralisé ou discours)

$\mathrm{Au}$ cours desquelles l'apprenant parle seul, sans interagir avec autrui. Ces activités impliquent une prise de parole de manière autonome et partent en général d'un déclencheur (textes, images, scènes vécues, etc.) qui peut l'inspirer et la stimuler.

- Exemples de ce type de la production:

- Narration (des histoires, des contes).

- Compte rendu (des expériences, des films).

- Récitation (des textes de théâtre, des poèmes).

- Élocution (sur des thèmes d'actualité, des problèmes de société).

- Description (des objets, des personnages).

- Exposé ou présentation.

B- Activités de la production orale en interaction (en face à face)

Elles se présentent comme spécificité d'être des situations quasiauthentiques, c'est-à-dire la simulation des situations réalistes (situation d'interlocution, appelée plus communément production dialoguée). 
- Exemples de ce type de la production:

- Conversations, dialogues, mises en commun et discussions.

- Improvisations.

- Débats, entretiens, simulations et entrevues.

- Cercles littéraires et réunions formelles.

- Question - réponse.

- Interviews.

- Négociations pour obtenir des biens et des services.

- Mini-enquêtes.

- Évaluation de la production orale Germain, Netten et Movassat (2004) soulignent que l'évaluation de la production orale a une place primordiale dans le processus d'enseignement/ apprentissage du FLE. Elle se fait en général au travers des grilles que les enseignants élaborent par rapport à des tâches plus ou moins difficiles que les apprenants doivent accomplir, et aussi au travers de l'estimation d'autres collègues. En outre, il existe trois approches pour évaluer la production orale et l'analyse du discours:

1. L'interaction entre le contexte et les acteurs.

2. L'interaction entre les acteurs.

3.L'observation de la force communicative verbale des acteurs.

D'autre part, Georges (2013) détermine trois aspects d'évaluation de la production orale:

\section{A. Linguistiques:}

Aspects de phonétique, de lexique, de grammaire ou de morphosyntaxique.

\section{B. Pragmatiques:}

Aptitude de transférer adéquatement les informations: La fluidité, les registres qui doivent être constants et appropriés aux situations et l'enchaînement (l'aptitude de remplir les vides, de reformuler, de comprendre les interlocuteurs et de leur répondre convenablement).

\section{Expressifs:}

Intonations expressives, emploi des images ou des figures de style (le discours devient plus vivant, efficace), la posture et la gestuelle qui doivent être normales et facilitation de la conception du message.

\section{Dispositif méthodologique de la recherche}

\section{- Choix de l'échantillon de la recherche}

Le choix d'un échantillon des élèves de la première année préparatoire aux écoles de langues revient à quelques raisons:

- On a appliqué une étude pilote à 43 élèves en première année préparatoire aux écoles officielles de langues à Mansourah afin d'identifier la problématique de la recherche. Les résultats de cette étude ont indiqué qu'il y a une faiblesse en compétences de la production orale auprès de ces élèves.

- Ces élèves n'ont pas appris le français en primaire. Ainsi, il y a une pauvreté lexicale, grammaticale et un manque de maîtrise phonologique chez eux qui expliquent leur inaptitude à parler.

- Ces élèves doivent avoir beaucoup d'occasions de prendre la parole en continu et en interaction dans la classe afin d'acquérir la langue française et de pratiquer l'oral spontanément et avec aisance.

- Il est nécessaire de développer les compétences de la production orale auprès de ces élèves à partir de la première année préparatoire. Cela va les aider durant les prochaines années à communiquer efficacement dans les différentes situations.

\section{- Instruments de la recherche}

1- Questionnaire des compétences de la production orale

$\mathrm{Ce}$ questionnaire vise à déterminer les compétences de la production orale nécessaires aux élèves de l'échantillon de la recherche. Il se compose de trente huit compétences incluses dans 


\begin{abstract}
cinq dimensions essentielles (la prononciation, le vocabulaire, la grammaire, la fluidité et le contenu). En vue de vérifier la validité de ce questionnaire, on l'a soumis à un jury spécialiste en didactique du FLE. Ces spécialistes ont avancé quelques propositions qu'on a prises en compte. Par ailleurs, d'après les points de vue du jury et le calcul du degré d'importance des compétences de la production orale, la chercheuse s'est limitée à développer les dix compétences les plus importantes et appropriées de la production orale auprès de l'échantillon de la recherche. (Annexe I)
\end{abstract}

\section{2- Test de la production orale}

Ce test ayant pour but de vérifier l'efficacité de l'unité proposée basée sur le brainstorming assisté par le multimédia pour développer quelques compétences de la production orale auprès de l'échantillon de la recherche. Il comporte six questions mesurant les compétences de la production orale auprès de cet échantillon: La première question concernant (la prononciation), la deuxième question concernant (la fluidité), la troisième question concernant (la grammaire), la quatrième question concernant (le vocabulaire), la cinquième et la sixième question concernant (le contenu). (Annexe II)

\section{- Étude pilote du test}

La chercheuse a appliqué l'étude pilote $\mathrm{du}$ test à un échantillon qui comporte 43 élèves en première année préparatoire aux écoles officielles de langues à Mansourah.

\section{- Fidélité du test}

Afin de vérifier la fidélité du test, la chercheuse a d'abord ré-appliqué le même test après un mois au même échantillon, puis elle a calculé le coefficient de corrélation des notes d'élèves dans les deux applications au moyen de l'équation de Pearson:
$\mathrm{R}=$

$\frac{N \sum X Y-\sum X \sum Y}{\sqrt{\left[N \sum X^{2}-\left(\sum X\right)^{2}\right]\left[N \sum Y^{2}-\left(\sum Y\right)^{2}\right]}}$

$\mathrm{R}=$ Coefficient de corrélation.

$\mathrm{N}=$ Nombre d'élèves.

$\sum=$ Somme.

$\mathrm{X}=$ Notes d'élèves dans la première application.

$Y=$ Notes d'élèves dans la deuxième application.

$\mathrm{X}^{2}=$ Carrés des notes d'élèves dans la première application.

$\mathrm{Y}^{2}=$ Carrés des notes d'élèves dans la deuxième application.

$$
\mathrm{R}=\frac{1954}{2400.80}=0.81
$$

Le coefficient de fidélité $=0.81$.

D'après cette valeur élevée, le test est fidèle.

\section{- Validité du test}

Afin de vérifier la validité du test, la chercheuse a suivi les procédures suivantes :

1- Présenter ce test à un jury spécialiste en didactique du FLE. Il a confirmé que ce test est valide.

2- Calculer la validité selon l'équation suivante:

$$
\begin{aligned}
& \text { La validité }=\sqrt{\text { la fidélité }} \\
& \text { La validité }=\sqrt{0.81}=0.90
\end{aligned}
$$

D'après cette valeur élevée, le test est valide.

\section{- Durée du test}

On a calculé la durée du test à travers l'équation suivante:

Durée du test $=$

Somme du temps consommépar chaque élève pour tous les membres du groupe

Durée du test $=\frac{3655}{43}=85$ minutes.

Alors, la durée du test convenable est 85 minutes.

3- Rubrique de notation pour le test de la production orale (scoring rubric for speaking test)

Cette rubrique ayant pour but 
d'évaluer avec le test la performance orale des élèves de l'échantillon de la recherche. Elle comporte cinq critères d'évaluation détaillés (la prononciation, le vocabulaire, la grammaire, la fluidité et le contenu) concernant les dix compétences de la production orale et quatre niveaux de qualité: locuteur avancé (4 notes), bon locuteur (3 notes), locuteur satisfaisant (2 notes), pauvre locuteur (1 note). (Annexe III)

\section{- Fidélité de la rubrique}

Pour vérifier la fidélité de la rubrique, la chercheuse et l'enseignante du français (qui a enseigné l'unité proposée à l'échantillon de la recherche) l'ont appliquée simultanément et indépendamment avec le test de la production orale à 43 élèves en première année préparatoire aux écoles de langues à Mansourah, puis la chercheuse a calculé le coefficient de concordance entre les deux évaluateurs par l'équation de Cooper:

Coefficient concordance $=$

Nombre de fois d'accord

Nombre de fois d'accord + Nombre de fois de désaccord $\times 100$

Coefficient de concordance $=\frac{9}{9+1} \times 100=90 \%$

D'après cette valeur élevée, la rubrique est fidèle.

\section{- Validité de la rubrique}

En vue de vérifier la validité de cette rubrique, on l'a soumise à un jury spécialiste en didactique du FLE. Ces spécialistes ont confirmé qu'elle est valide.

\section{- Contenu de l'unité proposée}

L'unité proposée vise à développer les compétences de la production orale auprès des élèves de l'échantillon. Elle se compose de trois séances d'enseignement et une séance de révision. Cette unité a des objectifs, des résultats d'apprentissage visés, les formes $\mathrm{du}$ brainstorming et les composantes multimédias utilisées, la démarche de l'enseignement d'après le brainstorming assisté par le multimédia et les consignes d'apprentissage.
En ce qui concerne les activités de chaque séance à l'unité, la chercheuse a proposé ces activités en ayant recours à des différentes composantes multimédias (textes illustrés, images, bandes dessinées, CDs, chansons, vidéo-clips, data-show, ordinateur, une affiche colorée concernant les règles du brainstorming) par lesquelles elle a exécuté le processus du brainstorming afin de développer les compétences de la production orale auprès des élèves de la première année préparatoire aux écoles de langues. De plus, la chercheuse a soumis l'unité proposée à un jury spécialiste en didactique du FLE. Ce jury l'a appréciée et elle a pris en compte ses suggestions. (Annexe V)

\section{- Guide pédagogique}

Le guide pédagogique aide l'enseignant du FLE aux écoles de langues à enseigner l'unité proposée d'après le brainstorming assisté par le multimédia en vue de développer les compétences de la production orale auprès des élèves de la première année préparatoire.

$\mathrm{Ce}$ guide contient une lettre adressée à l'enseignant incluant l'objectif, les éléments du guide et la philosophie du brainstorming et du multimédia sur laquelle l'enseignement de l'unité proposée porte. Il se compose aussi de quelques consignes que l'enseignant doit mettre en considération lors d'utilisation du brainstorming assisté par le multimédia dans la classe du FLE, des objectifs, des résultats d'apprentissage visés de l'unité proposée, le contenu de l'unité, les formes $\mathrm{du}$ brainstorming et les composantes multimédias utilisées, la démarche de l'enseignement d'après le brainstorming assisté par le multimédia, les consignes d'apprentissage, le plan temporel de l'enseignement de l'unité proposée et l'évaluation.

Après avoir préparé ce guide pédagogiue, on l'a soumis à un jury spécialiste en didactique du FLE afin de 
vérifier sa validité pour l'enseignement de l'unité proposée. Les membres du jury ont avancé quelques propositions qu'on a prises en compte. (Annexe IV)

\section{- Expérimentation}

Après avoir appliqué le pré-test le $6 /$ 2/ 2018, l'enseignement de l'unité proposée a eu lieu depuis 13 / 2/ 2018 jusqu'à 6/ 3/ 2018. L'expérimentation a duré 4 semaines à raison de deux cours par semaine (période). Chaque cours dure 40 minutes.

L'enseignante du français aux écoles officielles de langues à Mansourah a enseigné l'unité proposée au groupe de la recherche d'après le brainstorming assisté par le multimédia. Après avoir fini l'enseignement de l'unité proposée, on a appliqué le post-test le 13/ 3/ 2018 pour l'analyse et l'interprétation statistiques des résultats obtenus.

\section{Résultats de la recherche}

- Analyse statistique de données

- Afin de tester la première hypothèse, on a employé:

Le test (T) afin de vérifier qu'il existe une différence entre les moyennes des notes du groupe de la recherche au pré/post test de la production orale. Le tableau suivant montre les résultats obtenus:

Tableau No.2

Résultats du groupe de la recherche au pré / post test de la production orale

\begin{tabular}{|c|c|c|c|c|c|c|c|}
\hline Dimension & Test & M & ÉT & DL & $\mathbf{T}$ & $\mathbf{S}$ & $\eta^{2}$ \\
\hline \multirow{2}{*}{ Prononciation } & Pré & 2.1915 & .87572 & \multirow{12}{*}{46} & \multirow{2}{*}{22.3} & \multirow{12}{*}{0.05} & \multirow{2}{*}{0.92} \\
\hline & Post & 6.6170 & .99024 & & & & \\
\hline \multirow{2}{*}{ Vocabulaire } & Pré & 2.7872 & .83239 & & \multirow{2}{*}{14.4} & & \multirow{2}{*}{0.82} \\
\hline & Post & 5.5319 & .92903 & & & & \\
\hline \multirow{2}{*}{ Grammaire } & Pré & 2.6383 & .79196 & & 126 & & \multirow{2}{*}{0.78} \\
\hline & Post & 5.7234 & 1.17403 & & 12.0 & & \\
\hline \multirow{2}{*}{ Fluidité } & Pré & 2.7447 & 1.05229 & & \multirow{2}{*}{14.01} & & \multirow{2}{*}{0.81} \\
\hline & Post & 6.0426 & 1.06235 & & & & \\
\hline \multirow{2}{*}{ Contenu } & Pré & 5.8936 & 1.92505 & & \multirow{2}{*}{11.8} & & \multirow{2}{*}{0.75} \\
\hline & Post & 9.3830 & 1.22569 & & & & \\
\hline \multirow{2}{*}{ Note totale } & Pré & 16.2553 & 3.98076 & & \multirow{2}{*}{19.7} & & \multirow{2}{*}{0.89} \\
\hline & Post & 33.2979 & 3.79310 & & & & \\
\hline
\end{tabular}

M: Moyenne des notes.

ÉT: Écart-type.

DL: Degré de liberté.

T: Valeur de T.

S: Signification.

$\boldsymbol{\eta}^{2}$ : Éta carré.

Ce tableau indique qu'il existe une différence statistiquement significative au niveau 0.05 entre les moyennes des notes d'élèves du groupe de la recherche à la pré/post application du test de la production orale en faveur de la postapplication. En conséquence, la première hypothèse a été acceptée.

Ce tableau indique aussi que les valeurs de la taille d'effet $\left(\eta^{2}\right)$ dans toutes les dimensions sont élevées, en particulier la valeur concernant la prononciation (0.92), de même celle concernant la note totale est élevée (0.89).

- Afin de tester la deuxième hypothèse, on a calculé:

- La proportion du gain modifié de Black et la proportion d'efficacité de McGogian pour vérifier l'efficacité de l'unité proposée basée sur le brainstorming 
assisté par le multimédia pour développer quelques compétences de la production orale. Le tableau suivant montre les résultats obtenus:

Tableau No.3

Proportion du gain modifié de Black et proportion

d'efficacité de McGogian

\begin{tabular}{|c|c|c|c|c|}
\hline $\mathbf{N}$ & $\mathbf{M N P r}$ & $\mathbf{M N P o s}$ & $\mathbf{P G}$ & $\mathbf{P}$ \\
$\mathbf{T}$ & $\mathbf{e ́}$ & $\begin{array}{c}\text { t } \\
\mathbf{E}\end{array}$ & M & (16.3 \\
\hline 40 & 16.3 & 33.3 & 1.2 & 0.6 \\
\hline
\end{tabular}

NT: Note totale du pré/post test.

MNPré: Moyenne des notes du pré-test de la production orale.

MNPost: Moyenne des notes du post-test de la production orale.

PGM: Proportion du gain modifié de Black.

PE: Proportion d'efficacité de McGogian.

Ce tableau indique que les deux valeurs de la proportion du gain modifié de Black (1.2) et de celle d'efficacité de McGogian (0.6) sont équivalentes aux valeurs fixes (1.2) et (0.6).

Ces deux valeurs prouvent donc qu'il existe une efficacité de l'unité proposée basée sur le brainstorming assisté par le multimédia pour développer quelques compétences de la production orale auprès de l'échantillon de la recherche. En conséquence, la deuxième hypothèse a été acceptée.

\section{- Interprétation des résultats}

Les résultats de la recherche reviennent à quelques facteurs, comme:

- Mettre en pratique des diverses activités créatives de la production orale en continu et en interaction dont les élèves ont vu le sens et l'intérêt et qui centrent sur des situations de la vie quotidienne, a favorisé la véritable communication et la pratique de la production orale.

- Encourager toujours les élèves à s'exprimer spontanément et à parler entre eux et avec l'enseignant même s'ils ont commis des erreurs.
- Permettre aux élèves de choisir leurs partenaires et réduire le nombre d'élèves par groupe pendant le brainstorming collectif, cela a encouragé les élèves, surtout les timides à la prise de parole et à la participation orale.

- Utiliser le brainstorming a incité les élèves à exprimer leurs idées et leurs points de vue, à écouter et à respecter ceux d'autres, ainsi à critiquer de manière positive.

- Recourir à des moyens multimédias variés et appropriés, a créé le besoin et le désir de la communication orale auprès des élèves.

- Créer un environnement d'apprentissage qui a permis d'amener et de motiver les élèves à pratiquer l'oral et à apprendre d'une façon plus rapide et plus efficace à l'aide des nouvelles technologies et techniques.

- Fournir à l'enseignante un guide pédagogique qui a comporté tous les éléments nécessaires pour enseigner l'unité proposée en utilisant le brainstorming assisté par le multimédia dans la classe du FLE.

- Recommandations de la recherche recherche, on propose ces recommandations:

- La nécessité de l'utilisation des différentes formes du brainstorming en classe du FLE à tous les cycles éducatifs.

- La nécessité d'intégrer les technologies modernes dans le domaine de l'enseignement/ apprentissage du FLE.

- $\quad$ Prendre grand soin de faire parler les apprenants pour maîtriser la langue française en leur fournissant un bon modèle linguistique de qualité.

- Inciter les responsables de l'élaboration des programmes et des manuels scolaires à mettre en 
considération pendant leur planification les compétences orales, comme objet d'apprentissage à part entière.

- Organiser des ateliers en vue d'entraîner les enseignants du français sur l'utilisation des multimédias variés dans la classe.

- S'intéresser à évaluer d'une façon continuelle la performance orale chez les apprenants en ayant recours à des différents outils et méthodes de l'évaluation.

\section{- Suggestions de la recherche}

On propose quelques suggestions selon les résultats obtenus, comme:

- Utiliser le multimédia en vue de développer les compétences orales auprès des élèves du cycle primaire aux écoles distinguées de langues.

- Comparer l'effet d'utilisation du brainstorming et de la stratégie de six chapeaux de la réflexion sur le développement de la communicaton orale auprès des étudiants du cycle secondaire.

- Proposer une unité basée sur le multimédia pour développer l'achèvement et l'attitude envers la langue française auprès des étudiants du cycle secondaire.

- Proposer un programme basé sur le brainstorming électronique pour le développement des compétences de l'expression écrite auprès des futurs enseignants.

- Proposer un programme basé sur l'hypertextualité en vue de développer les compétences de la lecture auprès des élèves du cycle préparatoire.

\section{Bibliographie \\ - Références en langue française}

Abdella, N. (2001): Efficacité de la stratégie de l'apprentissage coopératif en vue de développer les compétences orales en langue française chez les élèves du cycle préparatoire, Thèse de Magistère non publiée, Faculté de Pédagogie, Université d'Hélouân.

Abdel-KHalek, E. (1998): L'expression du temps analyse de productions linguistiques (orales) d'égyptiens francophones, Thèse de Magistère non publiée, Faculté de Jeunes Filles, Université d'Ain-Chams.

Aycan, A. (2013): L'enseignement du FLE aux enfants assisté par le multimédia, Turkish Studies, Vol. 8, No. 10.

Bachelet, R. (2009): Comment animer un Brainstorming.

http://repo.mynooblife.org/Divers/gesti on projet/qualite brainstorming.

Barker, A. (1999): Brainstorming: comment libérer votre créativité en 30 minutes. Paris: Les Éditions JV \& DS.

Bakhit, O. (2017): Efficacité d'un programme basé sur la théorie des intelligences multiples pour développer certaines compétences orales chez les étudiants du cycle secondaire, Thèse de Magistère non publiée, Faculté de Pédagogie, Université de Mansourah.

Blanche- Benveniste, C. (2010): Approches de la langue parlée en français, Paris: Ophrys, $2^{\text {ème édition. }}$

Carrier, J-P. (2000): L'école et le multimédia. Paris: Hachette Éducation.

Charmeux, E. (2003): Apprendre la parole. Paris: Sedrap.

Delacroix, É. (2016) : Comment ne pas rater son brainstorming. Cerveau et Psychologie, No.78, Juin.

Delacroix, É. \& Galtier, V. (2005): Le groupe est-il plus créatif que l'individu isolé ? Le cas du brainstorming : 1953 - 
2003, cinquante ans de recherche, Revue Management et Avenir, Vol. 2, No. 4.

El- Battrawy, A. (2004): Programme suggéré pour développer les compétences orales en français pour les élèves des écoles du tourisme et d'hôtellerie en vue de leurs besoins fonctionnels, Thèse de Doctorat non publiée, Faculté de Pédagogie, Université du Canal de Suez.

Fournier, J. (2005): Scénarisation et multimédia: Processus de scénarisation interactive. Canada : Les Presses de L'université Laval, $2^{\text {ème }}$ édition.

Georges, S. (2013): Évaluer la production orale au travers d'une démarche scientifique. Revue Française de Linguistique Appliquée, Vol. 18, No. 1.

Germain, C., Netten, J. \& Movassat, P. (2004): L'évaluation de la production orale en français intensif: critères et résultats. Revue Canadienne des Langues Vivantes, Vol. 60, No.3

Giurgiu, I. (2013): Stratégies de production orale : étude d'un corpus de productions orales en interaction d'apprenants de français langue étrangère de niveau B2, Synergies Roumanie, No.8.

Guichon, N. (2006): Langues et TICE. Méthodologie de conception multimédia. Paris : Ophrys.

Hirschsprung, N. (2005): Apprendre et enseigner avec le multimédia. Paris: Hachette.

Izard, I. \& Cahn, N. (2012): Brainstorming box: Comment inventer en groupe de très bonnes idées. Paris: Eyrolles.

Kassas, B. (1997): Évaluation des compétences orales chez les enseignants $\mathrm{du}$ français au cycle préparatoire en Égypte, Thèse de Magistère non publiée, Faculté de Pédagogie, Université d'Ain-Chams.
Lafontaine, L. (2010): Activités de production et de compréhension orales. Présentation de genres oraux et exploitation de documents sonores. Montréal: Chenelière Éducation.

Leac, J-P. (2017): Le brainstorming ou remue- méninges. https://www.lescahiersdelinnovation.co m/2015/08/le-brainstorming/

Legros, D. \& Crinon, J. (2002): Psychologie des apprentissages et multimédia. Paris : Armand Colin.

Loiseau, Y. \& Roch, S. (2002): L'enseignant et la salle multimédia. FDLM, No.322, Juillet/Août.

Mesnards, P-H. (2012): Les 5 étapes incontournables d'un brainstorming efficace. $\quad$ www.techniquesingenieur.fr\%2Blivre blanc les-5etapes-dun-brainstormingefficace \%252520(2).\%26oq\&oq

Mohamed, S. (2000): L'efficacité d'utilisation de l'approche communicative sur le développement de skills de la conversation en français 1ère langue étrangère chez les élèves de la 2ème année préparatoire, Thèse de Magistère non publiée, Faculté de Pédagogie, Université de Tanta.

Ngouloure, J-P. (2013): L'expérience multimédia: De l'imaginaire global aux formes signifiantes. Paris: L'Harmattan.

Pacthod, A. \& Roux, P-Y. (1999): 80 fiches pour la production orale en classe de FLE. Paris : Didier.

Pothier, M. (2003): Multimédias, dispositifs d'apprentissage et acquisition des langues. Paris: Ophrys.

Riot, J. (2013): Le brainstorming, Revue Technologie, No.172, Juillet.

Roux, P-Y. (2003): L'oral en classe de langue: de la production à l'expression, FDLM, No. 327, Mai/Juin. 
Seguy, F. (1999): Les produits interactifs et multimédias. Grenoble: Presses Universitaires de Grenoble.

Sélim, KH. (1985): Développement de l'aptitude à parler la langue française chez les élèves du cycle préparatoire des instituts Azhariens, Thèse de Magistère non publiée, Faculté de Pédagogie, Université d'Al-Azhar.

Sorez, H. (2003): Prendre la parole. Expression écrite et orale. Paris: Hatier.

Stoebe, W. \& Nijstad, B. (2003): Le brainstorming en question. Cerveau et Psychologie, No.3, Septembre.

Swiners, J-L. \& Briet, J-M. (2004): L'intelligence créative audelà du brainstorming. Paris : Éditions Maxima.

Tomé, M. (2009): Productions orales, weblogs et projet de télécollaboration avec le web 2.0 pour l'enseignement du français (FLE). Revue ALSIC, Vol.12.

Youssef, A. (2003): Efficacité de quelques stratégies d'apprentissage sur le développement de certaines compétences auditives et orales chez les étudiants non-spécialistes, section de français, à la lueur de l'approche communicative, Thèse de Magistère non publiée, Faculté de Pédagogie, Université d'Hélouân.

- Références en langue anglaise

Agustina, E. \& Kumalarini, TH. (2014): "Doll steps" as a brainstorming game to improve the speaking skill in procedure text of the ninth graders of SMPN I Mojokerato. RETAIN, Vol. 2, No. 2.

Ampa, A., Rasyid, M. \& Basri, M. et al. (2013) : The implementation of multimedia learning materials in teaching English speaking skills. IJELE, Vol. 1, No. 3.

Asnawi, A. (2015): The effects of immersive multimedia learning with peer support on speaking skill among male and female students. SIELE Journal, Vol. 2, No. 2, September.

Borras, I. (2003): Developing and assessing "practicing spoken french": A multimedia program for improving speaking skills. ERIC, EJ478003.

Cullen, B. (1999): Brainstorming before speaking tasks. The Internet TESL Journal, Vol. 4, No. 7, July.

Diyyab, E. (2013): Using a multimediabased program for developing student teachers' EFL speaking fluency skills, Master's Thesis, Faculty of Education, Benha University.

Ghaemi, F. \& Hassannejad, E. (2015): Developing EFL students' speaking: Brainstorming VS. Role-Play. IJLLALW, Vol. 8, No. 4, April.

Hamideh, A. \& Rostampour, M. et al. (2015): The role of brainstorming in enhancing iranian EFL learners' speaking progress and achievement. MJLTM, Vol.5, No.2, June.

Khodadady, E., Shirmohammadi, S. \& Talebi, F. (2011): Brainstorming and its effect on critical thinking and speaking skills. Iranian EFL Journal, Vol.7, No. 1, February.

Liu, Y- CH. (2102): On fossilization of non-English majors' speaking ability in multimedia-assisted English class. IPCSIT, Vol.43.

\section{- Références en langue arabe}

$$
\begin{aligned}
& \text { صــلاح الــدين محمــود عــلام (0. . ب): } \\
& \text { الاسـاليب الاحصـائية الاسـتـلالية فـى } \\
& \text { تحليل بيانات البحوث النفسية و التربويـة }
\end{aligned}
$$

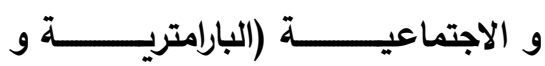

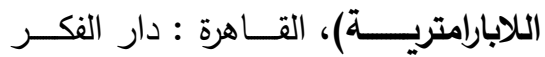

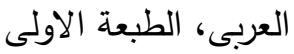

